\title{
Biosynthesis of Silver Nanoparticles Using Chlamydomonas reinhardtii and its Inhibitory Effect on Growth and Virulence of Listeria monocytogenes
}

\author{
Farajollah Shahriari Ahmadi *, Abass Tanhaeian, Maziar Habibi Pirkohi \\ Department of Biotechnology and Plant Breeding, College of Agriculture, Ferdowsi University of Mashhad (FUM), Mashhad, Iran \\ ${ }^{*}$ Corresponding author: Farajollah Shahriari Ahmadi, Department of Biotechnology and Plant Breeding, College of Agriculture, Ferdowsi University of \\ Mashhad (FUM), Iran. Tel: +98-5138805725, Fax: +98-5138787430, E-mail: shahriari@um.ac.ir
}

Received: August 02, 2015; Revised: December 27, 2015; Accepted: January 03, 2016

Background: Biosynthesis of nanoparticles using microorganisms, enzymes, and plant extracts is regarded as an alternative to chemical methods. Microalgae appear to be an efficient biological platform for nanoparticle synthesis as they grow rapidly and produce large biomass at lower cost.

Objectives: The possibility of silver nanoparticles biosynthesisby freshwater green microalgae, Chlamydomonas reinhardtii, was evaluated. Furthermore, antibacterial properties of the synthesized nanoparticles were investigated via analysis of growth and toxin production of Listeria monocytogenes.

Materials and Methods: Silver nanoparticles were synthesized by incubating $47.5 \mathrm{~mL}$ of fresh $C$. reinhardtii culture with $2.5 \mathrm{~mL}$ of $200 \mathrm{mM} \mathrm{AgNO} 3$ solution for $48 \mathrm{~h}$. Characterization of the synthesized nano particles was performed by Transmission Electron Microscopy (TEM), Scanning Electron Microscopy (SEM), Energy Dispersive Spectrometry (EDS) and X-ray diffraction analysis (XRD). Concentration of biosynthesized silver nanoparticles was measured by high resolution ICP-OES spectrometer. Inhibitory effect of silver nanoparticles on L. monocytogenes growth was measured. Further, the expression of listeriolysin $\mathrm{O}$ was investigated by serial microdilution method and Real-Time PCR assay.

Results: Spherical silver nanoparticles with average size of about $10 \mathrm{~nm}$ were formed. The particles had inhibitory effects on bacterial growth and antagonist activity on the expression of listeriolysin $\mathrm{O}$.

Conclusions: $C$. reinhardtii has the potential to be used as an effective platform for production of silver and other nanoparticles. Silver nanoparticles had potent antibacterial properties.

Keywords: Biosynthesis; Chlamydomonas reinhardtii; Listeriosis

\section{Background}

Metal nanoparticles with unique physicochemical properties have attracted much attention during recent years as useful element in electronics, medicine, textile, and sensing among many others (1).

Nanoparticles can be synthesized via a variety of techniques; amongst which, chemically synthesized nanoparticles are the most popular approach. Although chemical synthesis seems to be straightforward, the method poses threats to environment and well-beings of organisms (2). In most cases, chemical approaches employ toxic chemicals as reducing agents, organic solvents or non-biodegradable stabilizing agents. Since human are being exposed to noble metal nanoparticles such as gold and silver, development of safe and green processes of synthesis, free from toxic chemicals seems imminent (3).
Alternatives to chemical biosynthesis, being clean and cost effective to produce, have emerged using microorganisms, enzymes, and plant extracts (4). In between, microalgae, unicellular plants, including $C$. reinhardtii appear as the most suitable biological platforms for nanoparticle synthesis. The suitability of these organisms is due to their rapid growth and their potential in biomassproductionat lower cost (5).

Silver nanoparticles are mainly used as antimicrobial agents in textiles, wound dressings, and biomedical devices (6). Silver nanoparticles have been frequently tested for antimicrobial effects on a wide range of pathogenic bacteria (7).

The causative agent of listeriosis, Listeria monocytogenes is a Gram-positive facultative anaerobic bacterium found in a variety of food products, with devastating effect on human health (8). The pathogenesis of $L$. 
monocytogenes is mediated by so called virulence factors such as autolysin, positive regulatory factor $\operatorname{prf} A$, and the well-known toxin Listeriolysin $\mathrm{O}$. Listeriolysin $\mathrm{O}$ is a pore-forming thiol-activated cholesterol-binding cytolysi $\mathrm{n}$, which is encoded by hly gene (8).

The present study was conducted to evaluate the biosynthesis of silver nanoparticles by the $C$. reinhardtii and to evaluate the inhibitory effects of the particles on the growth and the expression of Listeriolysin $\mathrm{O}$ as the major virulence factor of $L$. monocytogenes.

\section{Objectives}

The main goal of the present study was to evaluate biosynthesis of silver nanoparticles using green microalgae $C$. reinhardtii. Proposing a green costeffective platform for production of nanoparticles is the main philosophy behind the present experiment. Moreover, since silver nanoparticles are believed to be of antimicrobial potential, the effect of the resulting nanoparticles was investigated on a harmful bacterial pathogen that is L. monocytogenes.

\section{Materials and Methods}

\subsection{Biosynthesis of Nanoparticles}

Healthy culture of $C$. reinhardtii was harvested in logarithmic phase and centrifuged at $2990 \times \mathrm{g}$ for 15 $\min$ at $4^{\circ} \mathrm{C}$. After discarding the supernatant, the biomass was washed with sterile water $3 \times$. The biomass was re-suspended in $47.5 \mathrm{~mL}$ of distilled water. $\mathrm{AgNO}_{3}(2.5 \mathrm{~mL}$ of the $200 \mathrm{mM})$ was added to microalgal biomass. Suspension of $C$. reinhardtii free of $\mathrm{AgNO}_{3}(50 \mathrm{~mL})$ was used as control. In all the experiments, $\mathrm{pH}$ was set at 8 . The experiment was carried out in triplicate and the cultures were incubated at $25^{\circ} \mathrm{C}$ for $48 \mathrm{~h}$.

\subsection{Characterization}

Color change in the culture was observed visually. The reduction of silver ions was monitored by measuring the absorbance of the reaction mixture in a range of wavelength from 300 to $500 \mathrm{~nm}$ using UV-Vis spectrophotometer. Morphology of the silver nanoparticles was studied using Transmission Electron Microscopy (TEM). TEM images were taken using Leo 912 AB high resolution transmission electron microscope operating at an accelerating voltage of $120 \mathrm{kV}$. A sample of the silver nanoparticles solution was placed on the carbon-coated copper grid and dried prior to microscopy. The biosynthesized silver nanoparticles [SNPs] were further studied by Scanning Electron
Microscopy (SEM) using LEO 1450VT microscope. For XRD measurement, a sample of silver nanoparticles solutionwas spread in a petri dish and oven dried. The dried sample was taken for XRD analysis using PHILIPS PW1480 X-ray diffractometer. Concentration of $0.2 \%(\mathrm{w} / \mathrm{v})$ biosynthesized SNPs was measured by high resolution ICP-OES spectrometer (SPECTRO ARCOS, Germany).

\subsection{Antibacterial Assay}

L. monocytogenes (RITCC 1624) was purchased from National Centre of Fungi and Bacteria, Iran. Minimum inhibitory concentration (MIC) of the biologically synthesized SNPs was determined using broth microdilution method in a 96-well standard ELISA plate. Luria Bertani (LB) broth containing $10^{5}$ CFU.mL ${ }^{-1}$ of $L$. monocytogenes cells was used as a culture medium. The final concentrations of SNPs were $0,12.5,25,50,100,200$ and $400 \mu \mathrm{g} . \mathrm{mL}^{-1}$. No organic solvent was used for dissolving SNPs, because such solvents possess their own inhibitory effect on bacteria. The medium was incubated in a shaking incubator at $37^{\circ} \mathrm{C}$ for $24 \mathrm{~h}$. The lowest concentration of SNPs inhibiting bacterial growth was assigned as MIC. To monitor growth dynamics of bacterial cells during exposure to SNPs, growth curve at 0,50 and $400 \mu \mathrm{g} . \mathrm{mL}^{-1}$ of silver nanoparticle was plotted via measuring optical density $(600 \mathrm{~nm})$ at different time intervals.

Real-Time PCR assay was conducted to assess expression of Listeriolysin O (hly) under SNPs treatment. RNA isolation and cDNA synthesis was conducted following general procedure (9). Primers were 5'-TTTCATCCATGGCACCACC-3' and 5'-ATCCGCGT GTTTCTTTTCGA-3'. Expression of the virulence gene (hly) was quantitatively analyzed using a Real-Time PCR system (Bio Rad). 16S rRNA gene was used as the calibration standard (9). Real-Time PCR was carried out in a $20 \mu \mathrm{L}$ reaction volume containing $0.5 \mu \mathrm{M}$ of each primer and $10 \mu \mathrm{L}$ of SYBR Green Real-time PCR master mix (Genet Bio, South Korea). Quantitative Real-Time PCR experiments were performed in duplicate for each sample.

\section{Results}

Color change was immediately observed after adding silver nitrate to $C$. reinhardtii suspension. The dark green color of $C$. reinhardtii was changed in to ivory at first and then to brown, indicating biosynthesis of silver nanoparticles. UV-Vis Spectroscopy, a surface plasmon resonance peak was observed at about $450 \mathrm{~nm}$, which 


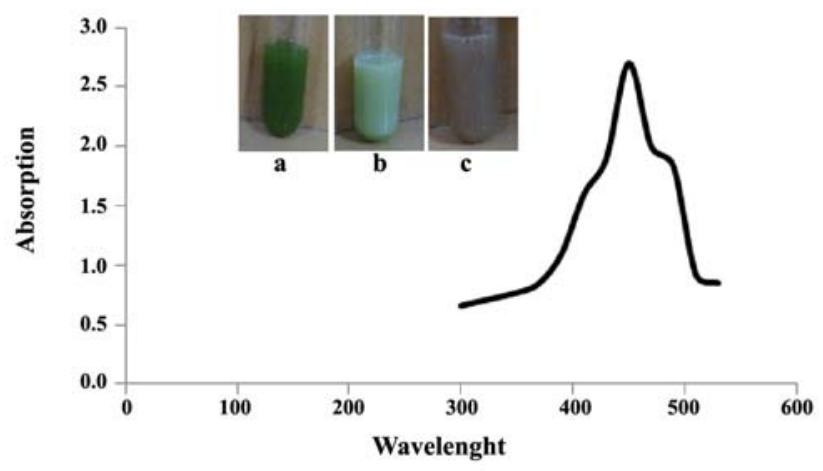

Figure 1. UV-absorption spectrum of Chlamydomonas reinhardtii suspension incubated with $\mathrm{AgNO}_{3}$. Intersect: color change in Chlamydomonas reinhardtii suspension indicating Bioreduction of silver ions into nanoparticles. (a: before addition of silver nitrate, b: immediately after addition of silver nitrate, c: after incubation period)

confirmed formation of SNPs (Figure 1).

Morphology of the biosynthesized SNPs was revealed by TEM microscopy (Figure 2). TEM image was analyzed using X software (4) (Table 1). The silver nanoparticles were about $10 \mathrm{~nm}$ spherical with circularity value $=1$.

Formation of silver nanoparticle was further confirmed by SEM imaging. Energy dispersive spectrometry (EDS) study indicated a sharp signal for Ag, confirming biosynthesis of silver nanoparticles. An absorption peak at $3 \mathrm{keV}$ confirmed presence of silver nanoparticles (Figure 3). The negligible peaks of $\mathrm{Au}$ and $\mathrm{C}$ in EDS graph weredue to impurities of the solution.

Diffraction properties and crystalline structure of the biosynthesized silver nanoparticles was characterized by X-ray powder diffraction. XRD results showed
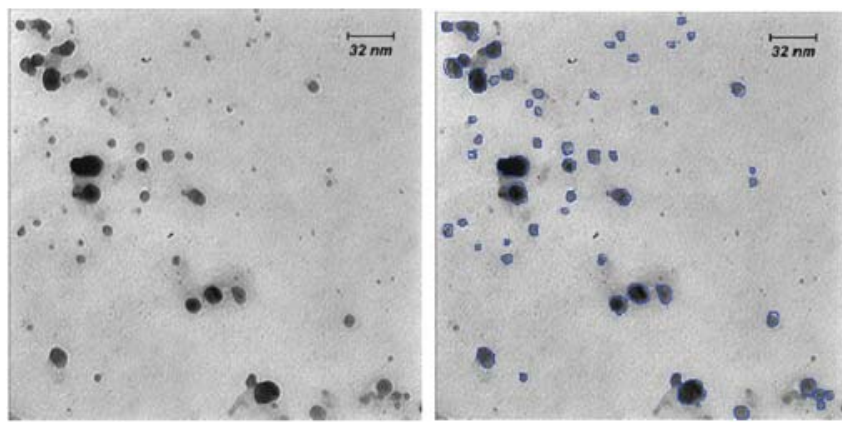

Figure 2. TEM micrograph of biologically synthesized silver nanoparticle (left). Monodispersed particles are highlighted by blue slots (right)

peaks corresponding to (111), (200), (220) and (322) Bragg reflections (Figure 4). This pattern clearly showed presence of SNPs in the sample. Based on XRD results, particle size can be calculated using Debye-Scherrer formula (2): $d=k \lambda / \beta \cos \theta$.

Where $d$ is the size of nanoparticle, $k$ stands for Scherrer constant (0.9), $\lambda$ represents the X-ray wavelength $(0.1541 \mathrm{~nm}), \beta$ is the full width at half maximum (FWHM), and $\theta$ is diffraction angle.

Using the Scherrer equation the average crystallite sizes of the SNPs was found to be in the range of 9-11 $\mathrm{nm}$; confirming particle size estimated by TEM images.

Concentration of the biosynthesized SNPs was determined using ICP method. Results showed that the concentration of SNPs in a $25 \mathrm{~mL}$ sample containing both SNPs and $C$. reinhardtii biomass was 2.934 mg. $\mathrm{L}^{-1}$ in average.

Serial microdilution was used to evaluate the inhibitory effect of SNPs on growth of L. monocyto-

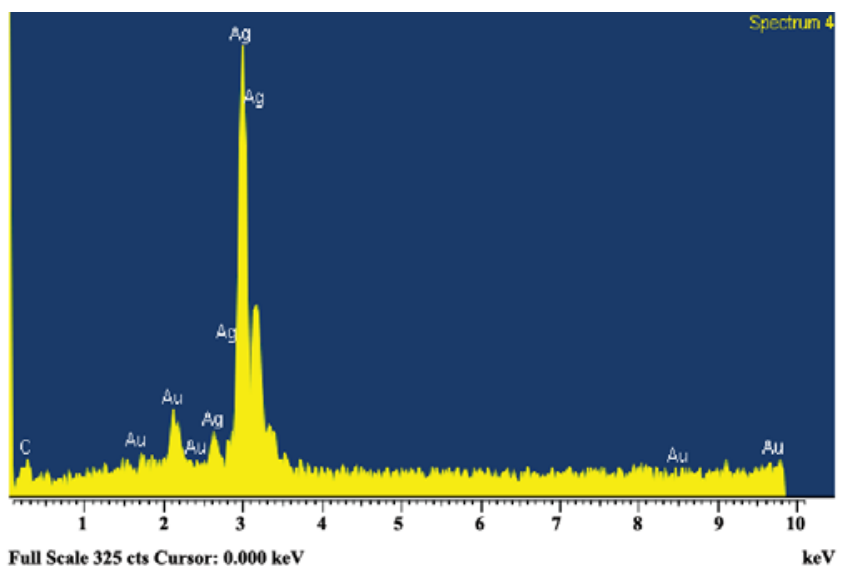

Figure 3. SEM micrograph of silver nanoparticles (left), EDS results indicating sharp peak for silver $\left(\mathrm{Ag}^{0}\right.$, right $)(\mathrm{X}=\mathrm{energy}$ level, $\mathrm{Y}=$ Absorption) 
Table 1. General properties of the SNPs biosynthesized using Chlamydomonas reinhardtii

\begin{tabular}{lc}
\hline Parameter & Average value \\
\hline Spherical diameter $(\mathrm{nm})$ & 10.95 \\
Width $(\mathrm{nm})$ & 6.05 \\
Length $(\mathrm{nm})$ & 7.38 \\
Roundness & 1.18 \\
Circularity & 1 \\
\hline
\end{tabular}

genes. Results of this test showed that SNPs at the concentration of $50 \mu \mathrm{g} . \mathrm{mL}^{-1}$ can inhibit growth of the pathogen; the minimum inhibitory concentration (MIC) of the SNPs was therefore determined as 50 $\mu \mathrm{g} . \mathrm{mL}^{-1}$. bacterial suspension showed normal growth below MIC value.

To further study the influence of SNPs on L. monocytogenes, growth kinetics of $L$. monocytogenes in a $12 \mathrm{~h}$ period was monitored under three concentrations of silver nanoparticles as 0 (control, non-treated), 50 $\mu \mathrm{g} . \mathrm{mL}^{-1}$ (MIC) and $400 \mu \mathrm{g} . \mathrm{mL}^{-1}$ (the highest concentration in this study). Growth kinetics graph of the bacterium under these SNPs treatments is presented (Figure 5). Control group showed a rapid growth pattern, which reached its maximum level about $12 \mathrm{~h}$ after culture. The treated bacterial sample with $50 \mu \mathrm{g} \cdot \mathrm{mL}^{-1}$ of SNPs manifested a decreasing growth pattern, so that after $10 \mathrm{~h}$ the growth level was nearly zero. Growth decreasing was more severe in the sample treated with $400 \mu \mathrm{g} \cdot \mathrm{mL}^{-1}$ of SNPs, so that a drop of growth was observed only after $4 \mathrm{~h}$, and the bacterial growth was completely inhibited $6 \mathrm{~h}$ post treatment.

Influence of SNPs on listeriolysin $\mathrm{O}$-as a virulence factor of L. monocytogenes was studied via Real-Time PCR (Figure 6). A dose-dependent decrease in expression of listeriolysin $\mathrm{O}$ under treatment with various concentrations of silver nanoparticles was recorded. In the range of 0 to $200 \mu \mathrm{g} \cdot \mathrm{mL}^{-1}$ of SNPs, a nearly linear relation was observed between SNPs concentration and reduction of listeriolysin $\mathrm{O}$ expression.

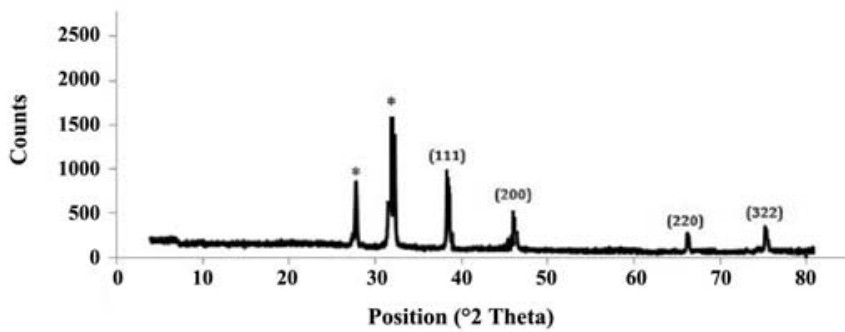

Figure 4. XRD pattern of silver nanoparticles biosynthesized by Chlamydomonas reinhardtii biomass

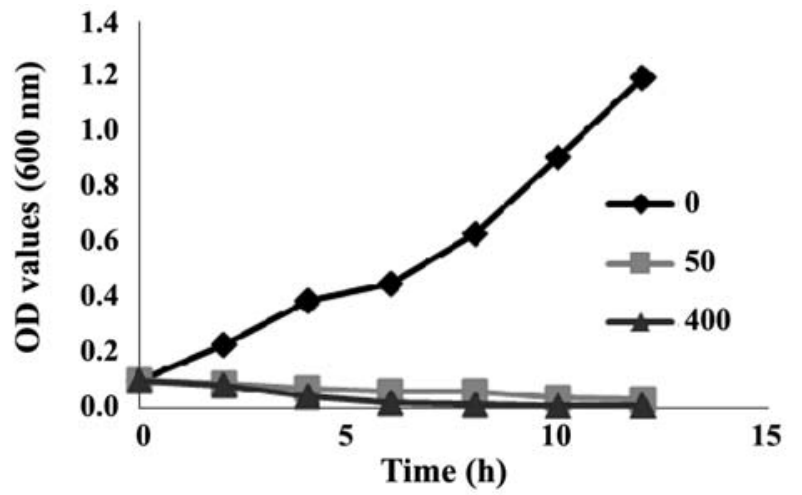

Figure 5. Growth kinetics of L. monocytogenes under treatment with different concentrations of biologically synthesized silver nanoparticles

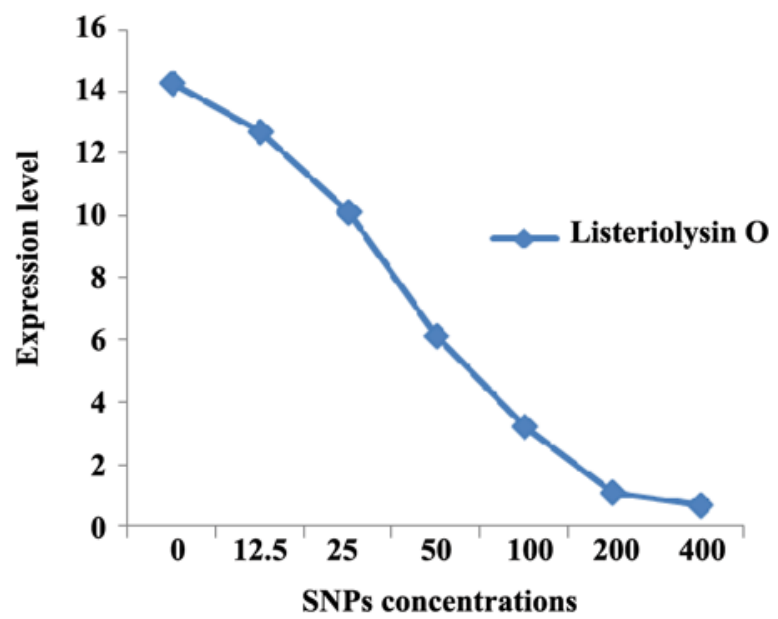

Figure 6. Quantification of listeriolysin O gene ( $h l y)$ expression in various concentrations of SNPs

\section{Discussion}

Biosynthesis of metal nanoparticle using a wide range of biological agents offers many advantages over conventional chemical and mechanical synthesis procedures. In the present study, biosynthesis of silver nanoparticles using suspension of $C$. reinhardtii was investigated. Microalgae are natural bio-remediators that due to their surface characteristics accumulate large amount of metal pollutants. During this bioaccumulation procedure, non-toxic metal containing compounds together with nanoparticles are generated from the trapped metal ions (10). C. reinhardtii is a green microalga, which naturally occurs in lakes and other fresh water sources (11).

The first indicator of bioreduction of Ag ions to silver nanoparticle is the characteristic change in color of the $C$. reinhardtii suspension. Some theoretical mech- 
anisms have been proposed to explain biosynthesis of nanoparticles by microalgae and $C$. reinhardtii cells. The most probable mechanism is the secretion of cellular reductases into the growth medium by $C$. reinhardtii cells. These enzymes can efficiently reduce silver ions in to silver nanoparticles (12). Moreover, metal ions can be trapped by the carboxylate groups residing on the surface of $C$. reinhardtii cells. The entrapped ions are then reduced by reductase enzymes, which subsequently results in the formation of nanoparticles (13). Presence of a maximum peak at about $450 \mathrm{~nm}$ in UV-Vis spectroscopy further confirmed biosynthesis of silver nanoparticles. Surface plasmon resonance peak in the range of $410 \mathrm{~nm}$ to 450 $\mathrm{nm}$ have been reported by other authors as an indicator of SNPs biosynthesis $(10,14)$.

Morphology of the biosynthesized SNPs was analyzed by TEM microscopy. Biologically synthesized nanoparticles can occur in various geometric forms. TEM images of the present study revealed that the biosynthesized silver nanoparticles were spherical with average diameter of $10.95 \mathrm{~nm}$ (Table1). Circularity of the biosynthesized SNPs was estimated to be 1, reconfirming spherical shape of the SNPs. Presence of SNPs in C. reinhardtii suspension was further confirmed by SEM images, EDS graph and XRD analysis. An absorption peak at $3 \mathrm{keV}$ in EDS study confirmed presence of silver nanoparticles in the solution. The $C$. reinhardtii biomass containing silver nanoparticles was dried and powdered for XRD analysis. Four peaks corresponding to (111), (200), (220) and (322) Bragg reflections were observed in this analysis. The XRD pattern obtained in this study was in accordance with previously determined Bragg reflections associated with silver nanoparticles $(15,7)$. Particle sizeswere estimated using XRD data and Debye-Scherrer formula indicating that the biosynthesized SNPs were 9-11 $\mathrm{nm}$ in average, which agrees with TEM microscopy results.

After characterization of SNPs, their antimicrobial effect on L. monocytogenes was studied by serial microdilution method. In vitro microdilution test showed that SNPs can inhibit the pathogen growth at concentration of $50 \mu \mathrm{g} \cdot \mathrm{mL}^{-1}$. Antimicrobial effects of silver nanoparticles have reported by many authors $(16,4,13)$. Silver nanoparticles produced in this study were about $10 \mathrm{~nm}$, which makes them of ideal size for inhibitory effects on bacterial cells. The size of nanoparticles plays critical role in their efficacy to inhibit microbial growth (17). It has been postulated that nanoparticles with smaller sizes have better antimicrobial effect, because they have larger surface area and higher percentage of interaction than bigger particles (16). Inhibitory effect of silver nanoparticles on bacterial growth can occur in many ways; for example, silver nanoparticles can interfere with sulfur containing biomolecules residing on the bacterial membrane, or they may attack bacterial genome and respiratory chain. These interfering effects ultimately result in bacterial cell death (18). In addition to investigating inhibitory effect of the SNPs on growth of L. monocytogenes, we evaluated their influence on expression of listeriolysin $\mathrm{O}$ as a major virulence factor of pathogen. Our results showed that SNPs, even at concentrations below MIC value, can reduce expression level of listeriolysin O. Negative effect of the biosynthesized SNPs on expression of virulence factors may offer medical implications in developing new antimicrobial medicines.

\section{Acknowledgements}

The authors wish to acknowledge the Ferdowsi University of Mashhad (FUM), Iran for financial support (Code 31416).

\section{Funding/Support}

The research was funded by department of Biotechnology and Plant Breeding, Faculty of Agriculture, Ferdowsi University of Mashhad.

\section{References}

1. Cui H, Feng Y, Ren W, Zen T, Lev H, Pan Y. Strategies of large scale synthesis of monodisperse nanoparticles. Rec Patents Nanotechnol. 2009;3:32-41. DOI: 10.2174/187221009787003302

2. Edison T, Sethuraman M. Instant green synthesis of silver nanoparticles using Terminalia chebula fruit extract and evaluation of their catalytic activity on reduction of Methylene Blue. Process Biochem. 2012;47:1351-1357. DOI: 10.1016/j.procbio.2012.04.025

3. Mittal A, Chisti Y, Banerjee U. Synthesis of metallic nanoparticles using plant extracts. Biotechnol Adv. 2013;31:346-356. DOI: $10.1016 /$ j.biotechadv.2013.01.003

4. Ali D.M, Thajuddin N, Jeganathan K, Gunasekaran M. Plant extract mediated synthesis of silver and gold nanoparticles and its antibacterial activity against clinically isolated pathogens. Colloids Surf B Biointerfaces. 2011;85:360-365. DOI: 10.1016/j.colsurfb.2011.03.009

5. Parial D, Patra H, Roychoudhury R, Dasgupta AK, Pal R. Gold nanorod production by cyanobacteria-a green chemistry approach. J Appl Phycol. 2012;24:55-60. DOI: 10.1007/s10811010-9645-0

6. Nowack B, Krug HF, Height M. 120 years of nanosilver history: Implications for policy makers. Environ Sci Technol. 2006;45:1177-1183. DOI: 10.1021/es103316q

7. Prasad TN, Kambala VSR, Naidu R. Phyconanotechnology: synthesis of silver nanoparticles using brown marine algae 
Cystophoramoniliformis and their characterization. J Appl Phycol. 2013;25:177-182. DOI: 10.1007/s10811-012-9851-z

8. Monnier AL, Abachin E, Beretti JL, Berche P, Kayal S. Diagnosis of Listeria monocytogenes Meningoencephalitis by Real-Time PCR for the hly Gene. J Clin Microbiol. 2011;49(11):3917-3923. DOI: 10.1128/JCM.01072-11

9. Bowman J, Bittencourt C, Ross T. Differential gene expression of Listeria monocytogenes during high hydrostatic pressure processing. Microbiology. 2008;154:462-475. DOI: 10.1099/mic.0.2007/ 010314-0s

10. Mehta SK, Gaur JP. Use of algae for removing heavy metal ions from wastewater: progress and prospects. Crit Rev Biotechnol. 2005;25:113-152. DOI: 10.1080/07388550500248571

11. Mahdieh M, Zolanvari A, Azimeea AS, Mahdieh M. Green biosynthesis of silver nanoparticles by Spirulinaplatensis. Scientia Iranica F. 2012;19(3):926-929. DOI: 10.1016/j.scient.2012.01.010

12. Kannan N, Subbalaxmi S. Biogenesis of nanoparticles-a current prospective. Rev Adv Mater Sci. 2011;27:99-114.

13. Mandal D, Bolander ME, Mukhopadhyay D, Sarkar G. Mukherjee P. The use of microorganisms for the formation of metal nanoparticles and their application. Appl Microbiol Biotechnol. 2006;69:485-492. DOI: 10.1007/s00253-0050179-3

14. Yasin S, Liu L, Yao J. Biosynthesis of Silver Nanoparticles by Bamboo Leaves Extract and Their Antimicrobial Activity. $J$ Fiber Bioeng Inform. 2013;6(1):77-84. DOI: 10.3993/jfbi 03201307

15. Dhanalakshmi PK, Riyazulla A, Rekha R, Poonkodi S, Thangaraju N. Synthesis of silver nanoparticles using green and brown seaweeds. Phykos 2012;42(2):39-45.

16. Rai M, Yadav A, Gade A. Silver nanoparticles as a new generation of antimicrobials. Biotechnol Adv. 2009;27:76-83. DOI: 10.1016/j.biotechadv.2008.09.002

17. Morones JR, Elechiguerra JL, Camacho A, Ramirez JT. The bactericidal effect of silver nanoparticles. Nanotechnology 2005;16:2346-2353. DOI: http://dx.doi.org/10.1088/0957$4484 / 16 / 10 / 059$

18. Sondi I, Salopek-Sondi B. Silver nanoparticles as antimicrobial agent: a case study on $E$. coli as a model for gram-negative bacteria. J Colloid Interface. 2997;275:177-182. DOI: 10.1016/j.jcis.2004.02.012 